\title{
Review Paper on Wireless Wearable Sensor System for Cardiac Activity Monitoring
}

\author{
Prof. G.K. Andurkar ${ }^{1}$, Ms. Jayashri Mohurley², Vishakha Borkar ${ }^{3}$ \\ Associate Professor, E\&TC, GCOEJ, Jalgaon, India ${ }^{1}$ \\ M. Tech Student, E\&TC, GCOEJ, Jalgaon, India ${ }^{2,3}$
}

\begin{abstract}
Ubiquitous vital signs sensing using wireless medical sensors are promising alternatives to conventional, inhospital healthcare systems. In this work, a wearable ECG sensor is proposed. This sensor system combined an appropriate wireless protocol for data communication with capacitive ECG signal sensing and processing. The entire system has small size, is thin, and has low power consumption compared to recent ECG monitoring systems. In addition, appropriate signal conditioning and processing were implemented to remove motion artifacts. The acquired ECG signals are comparable to ones obtained using conventional glued-on electrodes, and are easily read and interpreted by a cardiologist. Heart rate assessment, as well as heart rate variability parameters are computed in real time directly on the sensor, thus only a few parameters are sent via wireless communication for power saving. Hardware and software methods for heart beat detection and variability calculation are described and preliminary tests for the evaluation of the sensor are presented.
\end{abstract}

Keywords: ECG signals, Heart rate assessment, MEMS technology.

\section{INTRODUCTION}

Wearable systems for patients remote monitoring consist of three main building blocks:

1) the sensing and data collection hardware to collect physiological and movement data, 2) the communication hardware and software to relay data to a remote center, and 3) the data analysis techniques to extract clinicallyrelevant information from physiological and movement data. Recent advances in sensor technology, microelectronics, telecommunication, and data analysis techniques have enabled the development and deployment of wearable systems for patients' remote monitoring. Researchers have relied upon advances in the abovementioned fields to address shortcomings of ambulatory technologies that had previously prevented long-term monitoring of patients' status in the home and community settings.

The miniaturization of sensors and electronic circuits based on the use of microelectronics has played a key role in the development of wearable systems. One of the major hurdles to the adoption of sensing technology, especially for wearable applications, has been the size of the sensors and front-end electronics that, in the past, made the hardware to gather physiological and movement data too obtrusive to be suitable for long-term monitoring applications. Recent developments in the field of microelectronics have allowed researchers to develop miniature circuits entailing sensing capability, front-end amplification, microcontroller functions, and radio transmission. The flexible circuit shown in Figure 3.5 is an example of such technology and allows one to gather physiological data as well as transmit the data wirelessly to a data logger using a low-power radio. Particularly relevant to applications in the field of rehabilitation are advances in technology. MEMS technology has enabled the development of miniaturized inertial sensors that have been used in motor activity and other health status monitoring systems. By using batch fabrication techniques, significant reduction in the size and cost of sensors has been achieved. Microelectronics has also been relied upon to integrate other components, such as microprocessors and radio communication circuits, into a single integrated circuit thus resulting in System-on-Chip implementations [4].

Health monitoring applications of wearable systems most often employ multiple sensors that are typically integrated into a sensor network either limited to body-worn sensors or integrating body-worn sensors and ambient sensors. In the early days of body-worn sensor networks (often referred to as "body sensor networks"), the integration of wearable sensors was achieved by running "wires" in pockets created in garments for this purpose to connect body-worn sensors. An example of this technology is the system [8]. Such systems by design were not suitable for long-term health monitoring. Recently developed wearable systems integrate individual sensors into the sensor network by relying on modern wireless communication technology. During the last decade, we have witnessed tremendous progress in this field and the development of numerous communication standards for low-power wireless communication. Thisin turn will result in more effective healthcare delivery, both financially and therapeutically [8]. 


\section{LITERATURE SURVEY}

A. Introduction

In early 1960's, Kadish used a system, which includes several things namely glucose sensor, a processor and a pump to control glycerin in patients with diabetes [1]. To manage complex situations, the pump will need several MEMS based sensors to monitor more parameters like glucose, heart rate, temperature and ECG etc., [2]. Optical methods developed pertaining to sensing purpose became advantageous in biomedical field. In medical field, the opportunities offered by optical fibers are always advantageous [3]. Multi parameter constant vital signs monitors are available in intensive care setting since early 1960s. These medical devices are actually developed upon the introduction of microprocessor technologies in 1970s. Fortunately Moore's law concentrates on constant improvements in miniaturization and the low power needs of microprocessor that allows on-body wireless sensors [4].

Energy expenditure is the result of movement produced by skeletal muscles as per the physiological point of view [5]. The standard reference for physical activity is the energy expenditure [6]. In normal conditions of daily living, the measurement of energy expenditure is not possible and also impractical for population studies. Therefore the estimation of energy expenditure due to heart rate recordings, observations or movement registration is increasing. Due to the advancements in wireless technologies and energy-efficient design, new applications for wireless devices have been developed which includes remote monitoring, networked micro sensors etc [7].

In U.S.A itself, every year over 11 lakhs of people experience a heart attack. About 540,000 are fatal and approximately half of these deaths occur within intense care settings. Early detection and treatment of life threatening events can be done by monitoring the ECG continuously, thereby saving many lives [8]. In the year 1924, Einthoven received the Nobel Prize in physiology. Based on the observations made by Einthoven, traditional ECG medical instruments have been developed. During the experimental work, he placed the limbs of a subject in a salt solution connected to a string galvanometer. From that time, ECG signal have been observed on the monitoring devices. It is possible to interpretate multiple ECG channels by using the techniques of Microprocessors and Digital signal processing. As per Moore's law, Efficiency of ECG interpretation can be improved by applying the ever increasing computational power [8].

Equipment which combines small structures and electrostatic manipulation is referred as MEMS (Micro Electro Mechanical Systems) or NEMS (Nano Electro Mechanical Systems). MEMS or NEMS are referred as wide range of micro and nano machined devices which are not electromechanical [9]. Bio MEMS referred as the potential of MEMS and NEMS in biological applications. Wireless sensors and sensor networks are playing a vital role in scientific, technological and research fields.
Though sensor networks have been using since several years, the wireless applications brought drastic changes in the development of sensors. These sensors networks are different when compared with normal wireless networks and computer networks.

Many new researches are going on to design new sensors which make direct or indirect contact with human body to improve the quality of human life. So it rises more challenges to solve like limited energy, restricted life time etc., [10]. Many new researches are focused to develop quality of human life in terms of health by designing and fabricating sensors which are either in direct contact with human body or indirectly [11]. Using wireless sensor networks (WSN) in medical systems has become a major effort in recent years. But in most of these research tasks like signal data processing, health state decision making and urgent messages sending is done by using a remote server [12].

The monitoring of health using mobile computing, sensors and communication technologies can be termed as Mhealth. In past days, wireless monitoring involves measuring of physiological parameters namely heart beat, blood pressure, blood oximeter and physiological signals etc., [13]. Other signals include measuring of parameters like movement monitoring, fall detection, place tracking and other activities. The features of wireless networking are explained with different examples and applications [12].

The previous decade experienced a significant interest towards monitoring and sensing of health care. The demand for wireless connection increases with the advancement in biomedical sensors. This wireless sensor networks found applications in different fields. One of the main applications of sensor is its usage in wireless biomedical sensor network (WBSN). The main equipment used in WBSN is MSP430F1611 microcontroller with features like $10 \mathrm{~KB}$ of RAM, $48 \mathrm{~KB}$ of flash memory, 128 bytes of data storage and 8 channels of 12-bit analog to digital converter. WBSN employs IEEE 802.15.4 standard. Primary evaluation of WBSN involves development and integration of 3 lead ECG [16]. In 1992, L.G.linberg and others proposed a new method, which often uses fiber optic probe to monitor heart rate and respiration rate simultaneously [13]. In 1998, J.L. Kalju developed a system, which is capable of measuring different physiological parameters and are used to design a system for heart rate reconstruction for rate adaptive pacing [10]. In 2000, M. Nakagawara\& K. Yamakoshi introduced a portable instrument to monitor blood pressure, cardiac output and other cardiovascular variables [10]. In 2001, Loren Schwiebert, Sandeep K.S. Gupta and Jennifer Weinmann described the strength of smart sensors which are developed from the combination of sensing materials along with combined circuitry for other biomedical applications [12]. In 2001, Eugene Ingshawshih explained the use of wireless microsensors networks for medical monitoring and environmental sensing [12]. In 2002, Gentili G.B proposed a simple 
microwave technique to monitor the cardiac activity. This technique is dependent on changes in modulation envelope of amplitude modulated waves passing through the body [12]. Telemedicine would become an important health care procedure in future due to population increment and increment in treatment expenditure. There is huge number of communication techniques to transfer the data from a point of case to a hospital. But, it is unsuccessful when a patient needs to undergo continuous checkup and monitoring. YuM.Chi and gertcauwenberghs proposed a 3-lead wireless ECG device which uses a remote based WSN to transfer the measured heart rate signals from patient's body to a PC for monitoring and storage [12].

Various technologies are available to monitor patient's response continuously, which are low cost and made up of disposable sensors and electrocardiogram electrodes. These can be wearable on a human body. The wearable devices are embedded into an undershirt with smart shirt design. Their interconnection is made with data bus, which supports a variety of sensing devices. These devices are capable of communicating with sensors located at base of the shirt [12].

In the year 2004, Yuan-Hsiang Lin proposed a system which is a combination of PDA (Personal Digital Assistant) and WLAN (Wireless Local Area Network) for mobile patient monitoring [12]. In present days, every Medicare wireless system uses Bluetooth or Wi-Fi to communicate between sensors and monitor. These systems cannot work as network and the data collected from a sensor could be transferred to a monitor. In complicated medicare conditions, such as disaster, it is critical to gather medicare data to anyone device namely PDA, Laptop or PC. To overcome the above problems, in the year of 2009 , Wu Suyu and Yi Weidong proposed a system in which the information from number of nodes can be routed to a single receiving device. Two medicare sensors namely heart beat sensors and pulse oximeter are interconnected in a network. The parameters like heart beat and pulse oximeter monitoring devices uses a MSP430 microcontroller and is displayed on LCD screen, which are connected to WSN node via RS232 serial communication port [12].

In 2008, Peng Zhang and Ming Chen explained a remote healthcare system by using 89C51 MCU to monitor various physiological signals of patient [12]. Wan-Young Chung, Young-Dong Lee and Sang-Joong Jung described the development and design of wearable health care monitoring systems by using a built in electrocardiogram (ECG), accelerometer and oxygen saturation (SP02) sensors. The various devices namely ubiquitous sensor network (USN), wrist pulse oximeter and wearable chest leads are developed. Among them, the USN is built on MSP430 microcontroller. This USN collects the information from the sensors and the same information is sent to PC [12]. In 2009, Mohammed Mahdi Ahmed and Graham A.Jullien introduced the concept of wireless implantable microsystems to continuously monitor blood glucose levels. The microsystem consists of micro fabricated glucose biosensors attached to a transceiver chip. This chip receives $13.56 \mathrm{MHz}$ carrier and measures the concentration of glucose and sends information back to external reader via load-shift keying (LSK) [12]. Chao Chen and Carlos Pomalaza-Raez described the design of a wireless body sensors system to monitor patients daily activities at home. The human movements are selected by using accelerometer. These measured signals are transmitted wirelessly using an IEEE 802.15.4 complaint RF transceiver and recorded at a host system for post analysis. The measured data is sent to microcontroller after sampling. This data is read by C8051F353 microcontroller which includes a 24/16 bit ADC. The data is loaded into a computer via a cable and analyzed through software programs. Accelerometers are used as monitoring equipment in wearable sensor unit. This is used to resolve states such as standing, sitting and lying [30]. In 2009, S.S Sonavane, V.Kumar and B.P.Patil developed the design of low power and low cost wireless senor networks (WSNs) node using MSP430 and Nordiac nRF24L01. MSP430 microcontroller from Texas instruments was chosen to design WSN node. MSP430 uses only $2 \mu \mathrm{W}$ in sleep mode while maintaining RAM.

The nRF24L01 uses radio transceiver for license-free spectrum of $2.4 \mathrm{GHz}$ ISM band globally [13]. In 2009, Kang-Ming Chang and Shing-Hong Liu introduced the concept of portable sleep monitoring with the help of ECG and accelerator with Bluetooth transmission. A portable monitoring system in sleep mode depends on accelerator and ECG signals. This system consists of two sensors namely an ECG sensor and a tri-axis accelerator sensor and also consists of MSP430 microcontroller which is used for low-power analog to digital conversion. The remote monitoring and ECG are implemented in a lab view dependent interface [13].

A mobile phone is used to design a wrist-worn health monitoring terminal to measure the body's multiparameters. The mobile phone that is connected to this terminal has memory and display. It is a wireless radio terminal for communicating with the health service center. This terminal is made up of MSP430 microcontroller, electrodes to collect or to measure the signals and modules for signal processing. Wireless data transmission is done using bluetooth. It is used to measure the physiological signals [13].

In 2011, RemyaRavindran and Pradeep Kumar Jaisal developed a concept to measure ECG, temperature of a patient and wireless ZigBee data transmission. This wireless sensor belt is used to measure ECG, respiratory rate and skin temperature. In this mode, 8051 microcontroller and a ZigBee transceiver that supports IEEE 802.15.4 standard for the transmission of data were employed. It employs ZigBee technology that is very well suitable for the transmission of small amounts of data like sampled values of ECG signals. The advantages of ZigBee are low cost, less delay, low current consumption and capability to support huge number of nodes virtually. 
ZigBee is superior technology when compared to C. Cardiart 108T Digi

Bluetooth and WLAN [13].

Reza S.Dilmaghani in the year 2011 described the design of Wi-Fi sensor network that is capable of monitoring patient's chronic diseases at their home itself via a remote monitoring system. In present days, all types of monitoring systems transfer information regarding a patient to the hospital with the help of PCs located at patient's home. By using a remote monitoring wireless system and network nodes, usage of PC can be avoided. These nodes are connected to a central node via internet connection which is located at the hospital. The nodes of proposed wireless sensor networks are constructed using ECG sensors, MSP430 microcontroller, a CC2500 radio terminal and a simple network protocol. The captured signals are transmitted to an access point through wireless network which operates on $2.4 \mathrm{GHz}$ frequency. The access point is also a small device connected to internet via asynchronous digital subscriber line (ADSL). With the help of ADSL, the data is transmitted to the hospital through internet for real time analysis and storage [10].

B. Reveal LINQ ${ }^{\mathrm{TM}}$ Insertable Cardiac Monitor

- Powerfully Small.

- Medtronic company had madethis device.

- The Reveal LINQ ICM is the smallest heart monitor on the market.

- It automatically detects and records abnormal heart rhythms for up to 3 years.

- It's safe for use in an MRI setting

- The Reveal LINQ ICM is placed just under the skin of your chest in a simple outpatient procedure.

- The ultra-discreet heart monitor is not visible in most patients.

The revolutionary Reveal LINQ ${ }^{\mathrm{TM}}$ Insertable Cardiac Monitoring System may help your doctor diagnose and treat irregular heartbeats that may be related to unexplained fainting. It is designed for people who experience infrequent fainting symptoms.

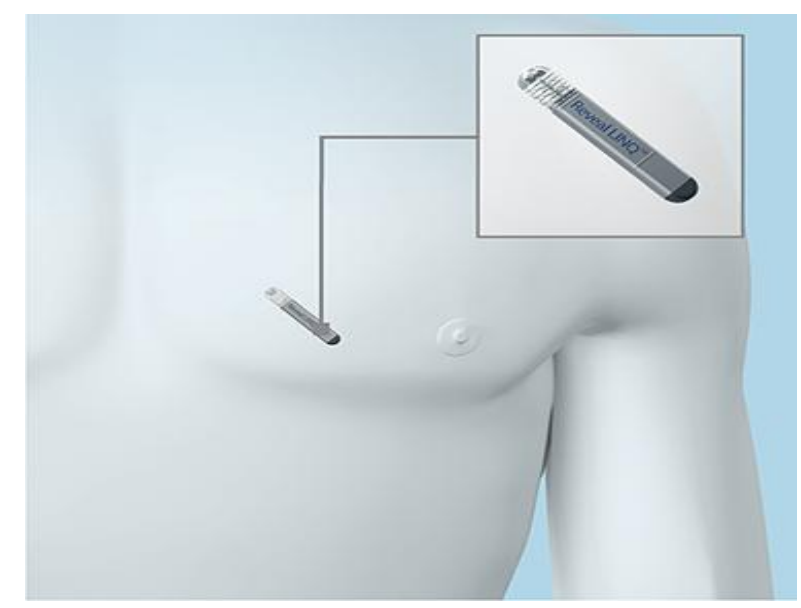

Fig.1Reveal LINQ ${ }^{\mathrm{TM}}$ Insertable Cardiac Monitor

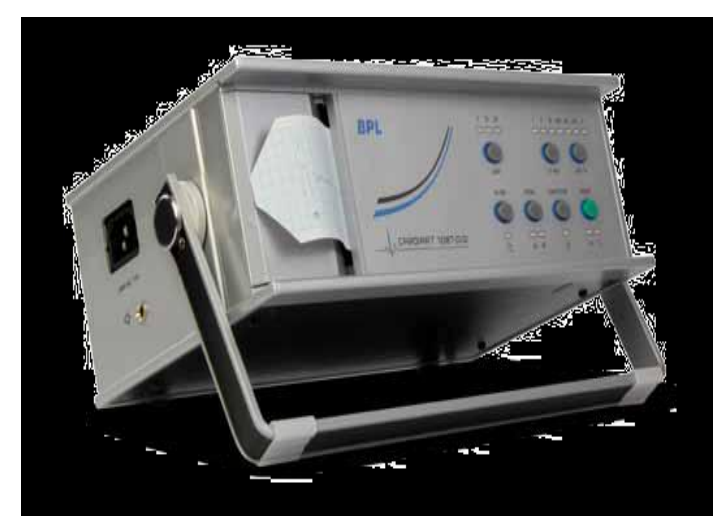

Fig.2Cardiart 108t Digi

- Single channel ECG recorder,

- Auto Power Off,

- Preserves the battery charge and activates automatically when not in use,

- Built-in Rechargeable Battery,

- Enable mains independent operations and provides battery use after charging,

- Defibrillation Voltage Protection,

- The machine is protected from defibrillators high voltage when used,

- Ease of Operation,

- The user-friendly design of the machine ensures easy operation,

- Real Time High Fidelity Thermal Array Recording,

- Enables the user to acquire accurate and quality recording,

- Electronic Lead Selection.

- Enables the easy selection of requisite leads for measurements,

- Lead off Indication,

- Notifies when one or more ECG electrodes are not properly affixed to a patient,

- Single Channel ECG Recording,Enables the recording of all leads sequentially.

\section{Proposed System}

This patient Monitoringsystem may reduce appointment time by allowing you to transmit data recorded by your cardiac monitoring system to your doctor from the comfort of home.

- This patient Monitoring system keeps you connected to your doctor with wireless, remote monitoring.

- Abnormal heart rhythm data is automatically sent to your doctor over a secure Internet connection.

\section{Reassuringly Easy}

- Uses a cellular signal and a power outlet. Setup is simple and easy.

- The clear, understandable animated display will show you exactly what to do.

- Go wherever you want. This system will go with you. 
The monitor must always be plugged in and in a location that receives an adequate cellular signal. For automatic transmission, you must be within $6.5 \mathrm{ft}(2 \mathrm{~m})$ of your monitor.

\section{CARDIACACTIVITYMONITORINGSYSTEM}

\section{A. Introduction to Cardiac Activity Monitoring System}

\section{1) Heart Rate Detection and Analysis:}

Heart related diseases kill more and people every year. The cost to detect and treat cardiovascular disease is too high. Monitoring and assessing the health of the heart for every cardiac cycle is of important and essential a wireless wearable sensorsystem for the continuous, long-term monitoring of cardiac activity. Heart rate assessment, as well as heart rate variability parameters are computed in real time directly on the sensor, thus only a few parameters are sent via wireless communication for power saving.

Hardware and software methods for heart beat detection and variability calculation are described and preliminary tests for the evaluation of the sensor are presented. With autonomy of Bluetooth Low Energy radio technology, this sensor will form apart of a wireless body network for the remote mobile monitoring of vital signals in clinical applications requiring automated collection of health data from multiple patients. The heart activity includes electrical signal (electrocardiogram or ECG) and mechanical actions. The electrical system is also called the cardiac conduction system. A graphical picture of the heart electrical activity is called electrocardiogram (ECG) [1]. Events in each heartbeat are very precise and highly coordinated.The system includes ECG sensor to collect electrical heart activity signals. Signals are then sent to a monitor using the Bluetooth radio technology. The monitor which connected to the network can take instruction from the healthcare personnel to take measurement, display, and store the heart activity waveforms heart status assessment. The set-up provides continuous monitoring if necessary or on-call basis when needed. Stored data can also be retrieved later for further assessment on the history change of the heart status.

\section{B. System Description}

This monitoring set-up includes a biosensor (i.e., a wearable device) to sense the heart activity and send the signals wirelessly to a smart phone to display and relay the signals to store in a network. ECG of the heart activityare collected at the same time to provide time events for the heart action assessment.

\section{1) Signal Sensing at Transmitter Side:}

The main sensors are an ECG circuit to detect ECG signal. The sensors are to be embedded in a wearable unit. The wearable device is to be worn on the patient chest. A microcontroller is used to take the signals from the ECG sensor, convert into digital form, and send the data to the Bluetooth module.

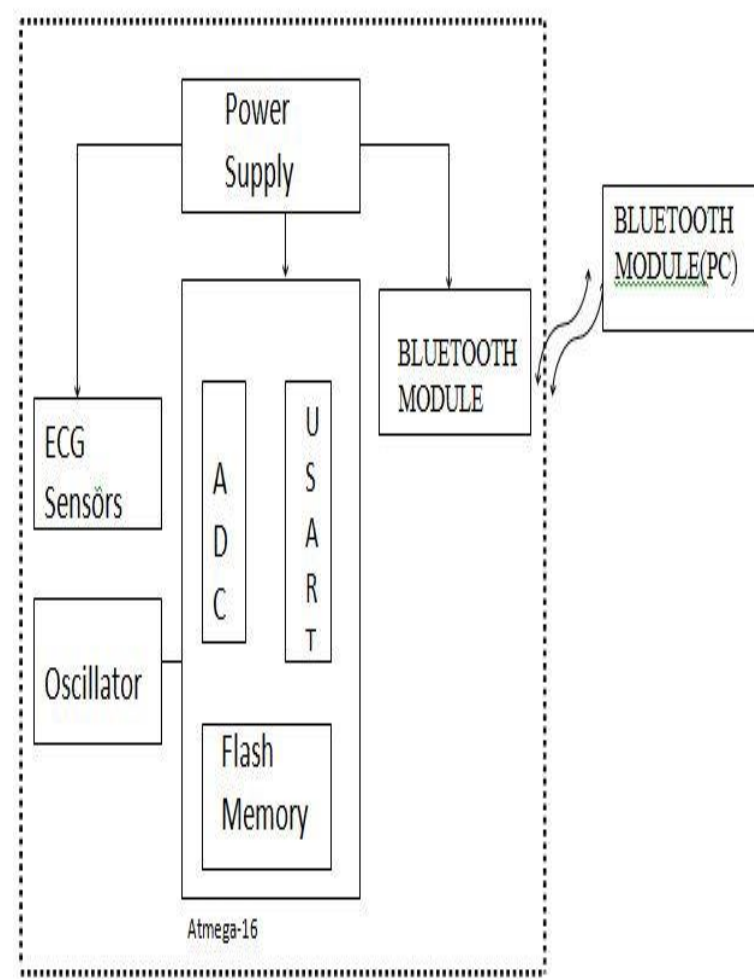

Fig 3.1 Basic Block Diagram of Proposed System.

A rechargeable battery (powersupply) is used to power the node. The weight and size of the node depend on the battery used. The use of the digital pulse is easier to calculate the heart rate and reducing transmitting power in wireless applications such as heart rate monitoring.

The traditional ECG sensor includes 3 electrodes (Left,Right, and Ground) and signal amplification circuit is shown and self-explained in Fig. 4. The application in the smart phone comprises of three modular threads: the Bluetooth transceiver module, data processing and plotter module, and the data up-loader module. The Bluetooth Transceiver module establishes a connection with the Bluetooth module in the sensor node to transmit appropriate control signals (packet) when needed. The application also sends an acknowledgement signal via serial data output back to the microcontroller via the Bluetooth module on successful data reception via Serial Data Input. The Data Processing and Plotter Module stores the data received from the BT module onto a queue which will then be plotted on the application using a suitable algorithm. The microcontroller converts the analog voltages of the sensors into digital form. The microcontroller sends data to the Bluetooth module using Rx-Tx(Receiver-Transmitter) Serial UART interface. The Bluetooth module manufactured by Roving Networks is used.

\section{Hardware Description}

The microcontroller usedhereisAtmega-16 that comes under AVR family which itself has an Analog to Digital Converter (ADC) converts the analog voltages of the 
sensors into digital form. The microcontroller then sends data to the Bluetooth module using $\mathrm{Rx}-\mathrm{Tx}$ (ReceiverTransmitter) Serial UART interface. The Bluetooth module manufactured by Roving Networks is used.

1) Power Supply Design:

Power supply is the first and the most important part of our project. For our project we require $+5 \mathrm{~V}$ regulated power supply with maximum current rating $500 \mathrm{~mA}$.

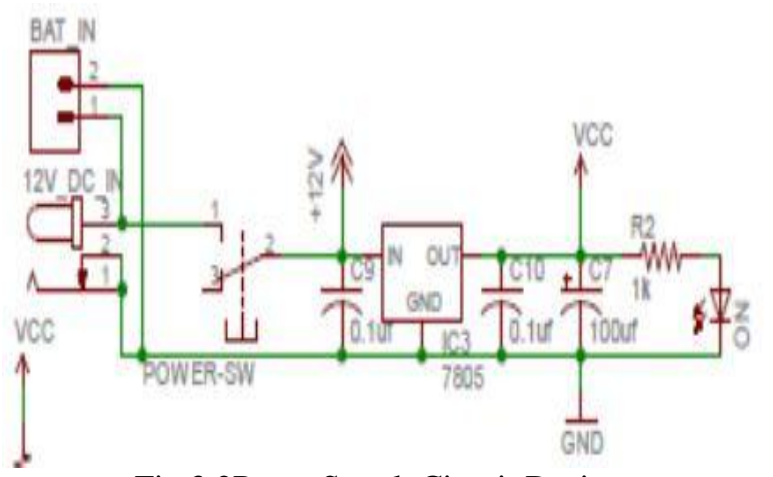

Fig.3.2PowerSupplyCircuit Design

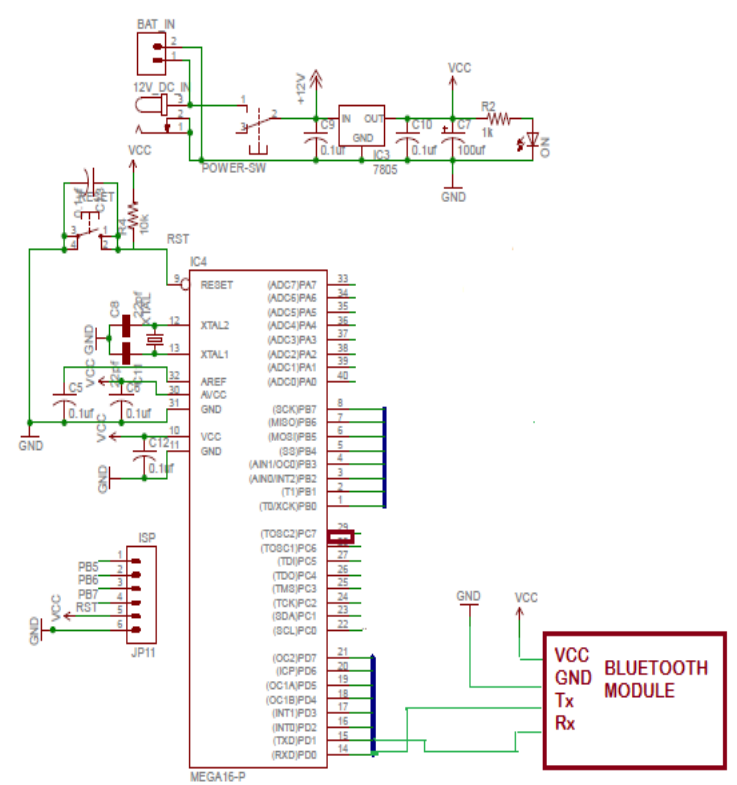

Fig 3.3ACircuit Diagram of Proposed System

\section{ECG Sensing System}

The electrocardiogram (ECG or EKG) is a diagnostic tool that is routinely used to assess the electrical and muscular functions of the heart. The electrocardiogram (ECG) has grown to be one of the most commonly used medical tests in modern medicine. Its utility in the diagnosis of a myriad of cardiac pathologies ranging from myocardial ischemia and infarction to syncope and palpitations has been invaluable to clinicians for decades. The ECG electrodes continuously tap the ECG signal from the patient's body. The raw ECG signal's amplitude lies in the range of $0.1 \mathrm{mV}$ to $2 \mathrm{mV}$ that needs to be amplified for further processing. Its frequency range is between $0.5 \mathrm{~Hz}$ to $250 \mathrm{~Hz}$. The instrumentation amplifier serves as a preamplifier with a gain of 50. This pre-amplified signal is fed to a high pass filter with a cut off frequency of $0.5 \mathrm{~Hz}$. It is then followed by a post-amplifier of gain 10 and a low pass filter of cut off frequency of $250 \mathrm{~Hz}$.

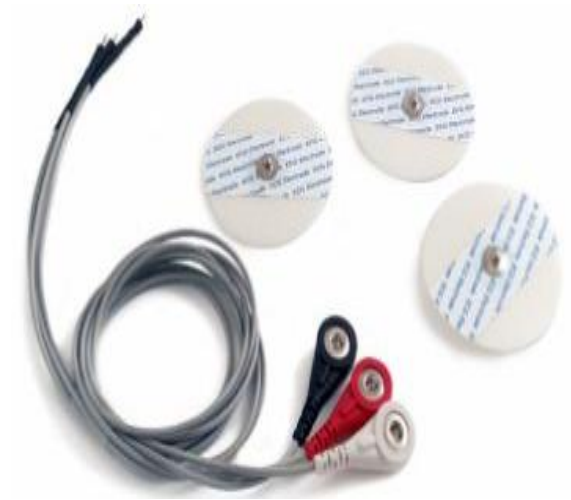

Fig.3.6ECG Sensors

1)General Description of ECG Sensor:

Heartbeats are triggered by bioelectrical signals of very low amplitude generated bya special set of cells in the heart (the SAnode). Electrocardiography (ECG) enables the translation of these electrical signals into numerical values, enabling them to be usedin a wide array of applications. Our sensor allow data acquisition not only at the chest ("on-the-person"), but also at the hand palms ("off-the-person"), and works bothwith propelled and most types of dry electrodes. The bipolar configuration is ideal for low noise data acquisition. The ECGsensorsareefficient due to the following features.

- Bipolar differential measurement

\section{Idle Mode}

When the SM2.0 bits are written to 000, the SLEEP instruction makes theMCU enter Idle mode, stopping the CPU but allowing SPI, USART, Analog Comparator, ADC, Two-wire Serial Interface, Timer/Counters, Watchdog, and the interrupt system to continue operating. This sleep mode basically halts clkCPU and clkFLASH, while allowing the other clocks to run.Idle mode enables the MCU to wake up from external triggered interrupts as well as internal ones like the Timer Overflow and USART Transmit Complete interrupts.This will reduce power consumptionin Idle mode. If the ADC is enabled, a conversion starts automatically when this mode is entered.
- Pre-conditioned analog output

- High signal-to-noise ratio

- Small form factor

- Raw data output

- Easy-to-use

- "On-the-person" and "off-the-person" use.

E. Bluetooth (IEEE 802.15)

Bluetooth (IEEE 802.15) is a universal short range low power radio protocol operating in the unlicensed industrial, scientific and medical frequency band. It allows both data and voice transmission. 


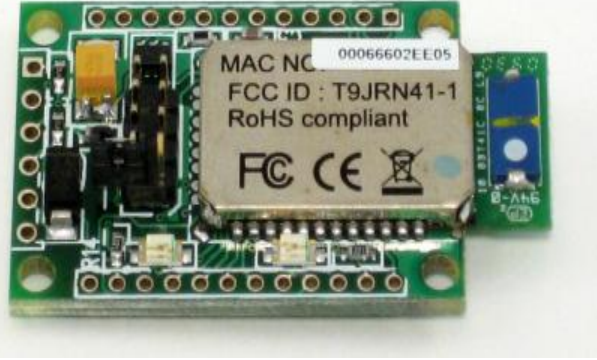

Fig3.7Bluetooth Module

The modulation technique is GSFK (Gaussian Frequency Shift Keying), with transmission at a rate of $1 \mathrm{M}$ symbols/s on one of 79 channels with $1 \mathrm{MHz}$ spacing in the $2.402 \mathrm{GHz}-2.480 \mathrm{GHz}$ band. Bluetooth uses the spreadspectrum frequency hopping connection with a rate of 1600 hops/s. Its key features are robustness, low complexity, low power and low cost. Compared to other methods of transmission, Bluetooth facilitates a noise free transmission. Moreover privacy and security options are highly advanced in the case of Bluetooth. We use BRC29 Blue Radios - 32 pin Bluetooth transceiver chip, which is a single chip solution for Bluetooth transmission. This chip requires an unregulated power supply of $1.9 \mathrm{~V}-3.4 \mathrm{~V}$ for its operation. Antennas need to be connected externally to the chip as shown in the Figure 3.7. The transceiver chip serves both as a transmitter and a receiver.

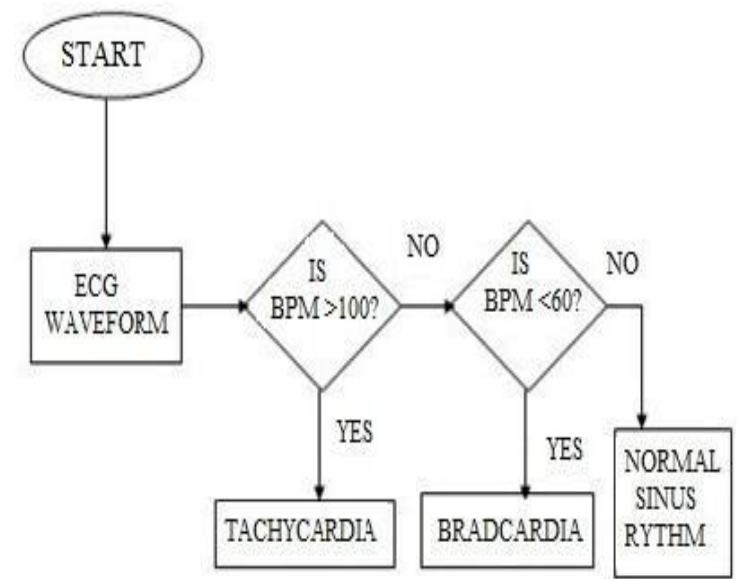

Fig 3.8 Flowchart Showing the logic of ECG Analysis

\section{CONCLUSION}

Monitoring systems that perform a complete ECG analysis in a local device near the patients are of great interest because they allow us to improve the quality of life for those who suffer from cardiac disorders. For 'an anywhere at any time monitoring system, devices used have to be actually mobile. All the components have been selected to design a light weight, easy to use, heart activity monitoring system .So that the ECG signals are to be sensed, processed, and sent to a PC for storage and display purpose. One of the problems in the detection of the ECG is the noise entering to the signal when the patient is in motion making the ECG waveforms undistinguishable.

Home care monitoring may have an important role as a strategy to provide effective and cost efficient health care for heart failure patients. The use of new improved technology to monitor patients along with the support of a health care provider significantly improves heart failure management while reducing the cost of health care. Additional multicenter, randomized controlled studies are required to further evaluate the potential benefits such as quality of life and cost effectiveness of these technologies and interventions.

\section{REFERENCES}

[1] S. Go, D. Mozaffarian, V. L. Roger, E. J. Benjamin, J. D. Berry, M. J. Blaha, S. Dai, E. S. Ford, C. S. Fox, S. Franco et al., "Heart disease and stroke statistics-2014 update: a report from the american heart association." Circulation, vol. 129, no. 3, p. e28, 2014

[2] http://www.nhlbi.nih.gov/health/dci/Diseases/hhw/hhw_electrical.h tml. Accessed on Feb. 14, 2011.

[3] Massot, N. Baltenneck, C. Gehin, A. Dittmar, and E. McAdams,"Emosense: An ambulatory device for the assessment of ans activity -application in the objective evaluation of stress with the blind," SensorsJournal, IEEE, vol. 12, no. 3, pp. $543-551$, march 2012.

[4] M. Altini, S. Polito, J. Penders, H. Kim, N. Van Helleputte, S. Kim,andF.Yazicioglu, "An ecg patch combining a customized ultra-lowpowerecgsoc with bluetooth low energy for long term ambulatory monitoring," in Proceedings of the 2nd Conference on Wireless Health. ACM, 2011, p. 15.

[5] Pantelopoulos and N. Bourbakis, "A survey on wearable sensorbasedsystems for health monitoring and prognosis," Systems, Man, andCybernetics, Part C: Applications and Reviews, IEEE Transactions on,vol. 40, no. 1, pp. 1-12, Jan 2010.

[6] K. Wac and C. Tsiourti, "Ambulatory assessment of affect: Survey ofsensor systems for monitoring of autonomic nervous systems activationin emotion," Affective Computing, IEEE Transactions on, vol. 5, no. 3,pp. 251-272, July 2014. [3] M. Stork, Z. Trefny. Electronic system for a Seismography measuring and signal processing. Applied Electronics International Conference, Sept 2005, Pilsen, Czech Republic.

[7] P. Bonato, "Wearable sensors/systems and their impact on biomedical engineering," IEEE Eng Med Biol Mag, vol. 22, pp. 1820, May-Jun2003

[8] R. Picard, "Future affective technology for autism and emotion communication," Philosophical Transactions of the Royal Society B:Biological Sciences, vol. 364, p. 3575, 2009.

[9] T. Gao, T. Massey, L. Selavo, D. Crawford, B. Chen, K. Lorincz, V.Shnayder, L. Hauenstein, F. Dabiri, and J. Jeng, "The advanced healthand disaster aid network: A light-weight wireless medical system fortriage," IEEE Trans Biomed Circuits Syst, vol. 1, p.

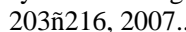

[10] M. Z. Poh, N. Swenson, and R. Picard, "A Wearable Sensor for Unobtrusive, Long-term Assessment of Electrodermal Activity," IEEE Trans Biomed Eng, vol. 57, pp. 1243-1252, 2010.

[11] X. Xiao, et al.,"Model-Based Assessment of Cardiovascular Healthfrom Noninvasive Measurements," Annals of Biomedical Engineering,vol. 30, pp. 612-623, 2002.

[12] H. Lea, J. L. Johnson and S. Ellingwood, "Telegenetics in Maine: Successful clinical and educational service delivery model developed from a 3-year pilot project", Genet Med, vol. 7, (2005), pp. 21-27.

[13] visithttp://nikomedusa.com

[14] M. K. Delano and C. G. Sodini, "A long-term wearable electrocardiogram measurement system," in 2013 IEEE International Conference on Body Sensor Networks, 2013, pp. 1-6. 